\title{
Membrane domains and the immunological synapse: keeping $T$ cells resting and ready
}

\author{
Michael L. Dustin \\ Department of Pathology and Skirball Institute of Biomolecular Medicine, \\ New York University School of Medicine, 540 First Avenue, New York, New York 10016, USA. \\ Phone: (212) 263-3207; Fax: (212) 263-3208; E-mail: dustin@saturn.med.nyu.edu. \\ J. Clin. Invest. 109:155-160 (2002). DOI:10.1172/JCI200214842.
}

The immune system has a huge stake in keeping mature $\mathrm{T}$ lymphocytes in a resting state until needed. Inappropriate $\mathrm{T}$ cell activation has devastating consequences for the host, including a variety of autoimmune diseases $(1,2)$. It is possible that the $\mathrm{T}$ cell maintains its quiescent state through overwhelming negative regulation, but this is unlikely because the $T$ cell has to be ready to react with great sensitivity to MHC-peptide complexes on the surface of the antigenpresenting cell (APC).

As few as three agonist MHC-peptide complexes on the surface of the APC are sufficient to activate a thymocyte for negative selection, whereas 300 are necessary to activate a naive $\mathrm{T}$ cell, with an intermediate number required for an effector T cell (3). Because only a fraction of these complexes will be found in the interface with a given thymocyte or $\mathrm{T}$ cell, it appears that thymocytes can be triggered by one MHC-peptide complex and that naive $\mathrm{T}$ cells require only about a dozen. Both thymocytes and mature $\mathrm{T}$ cells must measure interactions of $\mathrm{T}$ cell receptors (TCRs) with self-peptides that may be more numerous but that have interactions so weak as to be beyond reliable biochemical detection $(4,5)$. This high sensitivity indicates that $\mathrm{T}$ cells are poised to respond when only a few TCRs are perturbed. How is this high sensitivity achieved?

Another important aspect of $\mathrm{T}$ cell activation is that it must be sustained for a period of hours to program the $T$ cell for subsequent expansion and differentiation into effector cells. The shortest period of activation required for initiating this program in naive $\mathrm{CD} 8 \mathrm{~T}$ cells is 2.5 hours and in naive CD4 T cells is about 24 hours (6-8). The challenge for the $\mathrm{T}$ cells is to distinguish between very similar MHC-peptide complexes and to then integrate this process with innate immune responses over a period of hours. Clues to how the $T$ cells meet this challenge have been provided by studying the compartmentalization of signaling machinery and receptors in resting $\mathrm{T}$ cells and in $\mathrm{T}$ cells actively responding to physiologically presented MHC-peptide complexes. In this Perspective, I explore a number of recent findings that relate to the configuration of signaling components in the resting state and the conse- quences of perturbing this status quo to generate transient or sustained signals. I also consider the emerging role of membrane domains in integrating environmental information with signals from antigens, and I suggest a model for the recently described role of the ECM protein agrin in regulation of $\mathrm{T}$ cell responses.

The two-signal model

The current model for $\mathrm{T}$ cell activation breaks signaling into broad categories. Immunological specificity is provided by an initial signal, Signal 1, which is activated when the TCR recognizes processed antigen in the context of an MHC-peptide complex $(9,10)$. Innate immune responses that are essential to temper the response to antigen occur by means of a distinct signal, Signal 2, which is mediated by CD28 and various other coactivators and cytokine receptors $(11,12)$. Signal 1 could be considered the immunologically specific signal that alerts the host to the presence of a novel MHC-peptide complex, novel being defined by mature T cells whose previous experience is based on MHCpeptide complexes encountered in the thymus. The higher sensitivity of thymocytes to MHC-peptide complexes, relative to mature $\mathrm{T}$ cells, contributes to suppression of self-reactive $T$ cells and represents a margin of safety between thymic negative selection and mature $\mathrm{T}$ cell activation (3).

The immunological synapse

The TCR interactions with MHC-peptide take place in an intercellular junction, so the organization of the junction, both in the intercellular and lateral dimensions, is likely to be important for integration of antigen and innate cues. Because Signal 2 may also be received through an intercellular interaction, it is important to consider the nature of the intercellular junction in which these signals are processed. Recent insights into the molecular basis of this interface reveal a dramatic redistribution of signaling components to form an organized "immunological synapse," a term that borrows from Sherrington's turn-of-the-century coinage describing the grasping interconnections of neurons as synapses (13). In fact, the immunological 
synapse is one of a class of informational synapses that relay information across quasistable cell-cell junctions, others being the neuromuscular junction and many classes of CNS synapses.

The immunological synapse organizes and segregates adhesion molecules and TCR-associated components into two major compartments $(14,15)$. These areas, referred to as supramolecular activation clusters (SMACs), include the central (c) SMAC, which is enriched in TCRs, and the peripheral (p) SMAC, which contains lymphocyte function associated-1 (LFA-1) and talin (16). APC surface components are also integral to these clusters, such that MHC-peptide complexes are found in the CSMAC, whereas ICAM-1, the LFA- 1 counter-receptor, is concentrated in the pSMAC. Other APC specializations may exist, such as preclustered structures with class I and II MHC molecules and the CD28 ligand CD80 (17). The cSMAC also contains engaged CD28, while the PSMAC contains CD2 and LFA- 1 in segregated domains $(14,15)$. These are not homogenous structures, but they seem to be composed of smaller clusters that can sometimes be interspersed, as when LFA- 1 and CD2 penetrate the CSMAC region.

The immunological synapse develops over a period of minutes following initial interactions of the $\mathrm{T}$ cell and the antigen-presenting surface. Initially, the TCR is not necessarily engaged in the center, but as the cell-cell interaction develops, it translocates from the periphery into the center of the synapse. Formation of the synapse is concurrent with early TCR signals and depends upon an intact actin cytoskeleton, perhaps because actin polymerization and myosin-based contraction help localize the TCR and LFA-1 (18). It has also been proposed, based on mathematical models, that actin supports synapse formation by altering the physical properties of the membrane and the kinetics of the receptor-ligand interaction to favor the spontaneous self-assembly of synaptic components into structures of varying stability (19). The synapse is predicted to be stable when it includes agonist MHC-peptide complexes, but not antagonist MHC-peptide complexes. Intracellular transport mechanisms are also implicated in this process (20) and may serve to accelerate the formation of synapses, which may be stable over a period of hours, depending on their intrinsic features, such as receptor kinetics and arrangement and the physical properties of the membrane.

\section{Membrane rafts}

The organization of surface receptors and signaling components in the membrane and interactions with the cytoskeleton contribute to the balance of positive and negative factors that regulates TCR signaling. The first data to suggest that the fluid mosaic model had to be modified to incorporate lateral membrane domains were based on fluorescent dyes that reported differing degrees of order in the hydrophobic core of biological membranes (21). The concept of membrane domains was given biochemical substance by the observation that one class of domains enriched in cholesterol and sphingolipids is not fully soluble in nonionic detergents and can be isolated as a low-density fraction on density gradients. The finding that glycosyl phosphatidyl inositol-anchored proteins with apparent relevance to signaling processes are enriched in this fraction suggested that these domains are more than a biochemical curiosity (22). The current view is that these domains, or "rafts," are small regions of detergent-resistant liquid-ordered phase lipids in a bulk membrane with liquid-crystalline properties (23). Since the liquid-ordered phase is dependent upon cholesterol, agents like methyl- $\beta$-cyclodextrin, which can extract cholesterol from membranes, preferentially disrupt these domains. Cholesterol extraction results in a profound inhibition of phospholipase $\mathrm{C}$-triggered $\mathrm{Ca}^{2+}$ mobilization in $\mathrm{T}$ cells (24) but, paradoxically, activates phosphotyrosine signaling and the mitogenactivated protein kinase (MAPK) signaling pathway (25). Because full $\mathrm{T}$ cell activation requires triggering of the MAPK pathway, the $\mathrm{Ca}^{2+} /$ calcineurin/nuclear factor of activated $\mathrm{T}$ cells pathway, and the NF- $\mathrm{KB}$ pathway, however, $T$ cells do not become fully activated as a result of cholesterol depletion. Nevertheless, the partial signal that occurs under these conditions indicates an important role of rafts in maintaining the resting state of T cells. As discussed below, two regulatory circuits associated with rafts have been described that illustrate the roles of rafts in resting mature $T$ cells and in T cell activation.

\section{Rafts show self-control}

The common pathway for TCR-activated signaling involves a protein tyrosine kinase (PTK) cascade including members of three PTK families: the Src family, the Syk family, and the Tec family $(26,27)$. The Src family member Lck has a critical early role in triggering the cascade that then recruits the Syk family kinase ZAP-70 and the Tec family kinase Itk. The regulation of Lck is therefore critical for maintaining the resting state of the $T$ cell and for initiating the activation of signaling cascades. The recruitment of ZAP-70 to the TCR is dependent upon dually phosphorylated immunoreceptor tyrosine-based activation motifs (ITAMs; see Billadeau and Leibson, this Perspective series, ref. 28) in the cytoplasmic domains of the CD3 and TCR $\zeta$ chains. Additional signaling components are recruited through the adapter protein linker of activated T cells (LAT) (29). LAT is a palmitoylated transmembrane protein with a small extracellular domain and a large cytoplasmic domain with multiple tyrosines. Lck and Fyn are palmitoylated on their amino-terminus. The palmitoylation of LAT, Lck, and Fyn leads to concentration of these molecules in rafts. Lck regulation is interwoven with its localization, such that rafts can be seen to have a central role in both resting and activated $\mathrm{T}$ cell signaling.

The regulation of Src family PTKs has been illuminated by structural studies (refs. 30, 31; see also Hermiston et al., this Perspective series, ref. 32). Regulation revolves around two tyrosines: the activation loop tyrosine (394 in Lck) that is autophosphorylated to activate the kinase domain, and the C-terminal regulatory tyrosine (505 in Lck), which is phosphorylated by the C-terminal Src kinase (Csk) and interacts with 
the $\mathrm{SH} 2$ domain by an intramolecular interaction (33). A third regulatory locus is based on the intramolecular interaction of the $\mathrm{SH} 3$ domain with the connecting peptide between the SH2 domain and the kinase domain. When the C-terminal tyrosine is phosphorylated and the $\mathrm{SH} 3$ domain is engaged with the connecting peptide, the kinase is held in a "closed" conformation in which it is relatively inactive and its activation loop is unphosphorylated ( $\mathrm{Lck}^{\dagger}$ in Figure 1). Removal of the phosphate from tyrosine 505 by the transmembrane tyrosine phosphatase CD45 partially activates Lck, and further activation can be achieved by high concentrations of ligands for the $\mathrm{SH} 3$ domain that together "open" the conformation of the kinase $(34,35)\left(\mathrm{Lck}^{*}\right.$ in Figure 1). Therefore, the regulation of Lck depends, in part, upon the relative local activities of Csk and CD45.

Because CD45 can also dephosphorylate the activation loop tyrosine, it may be necessary for the cell to keep this phosphatase in a distinct compartment, away from sites of Lck activity (36). Indeed, CD45 is excluded from rafts and probably only encounters Lck at the boundary between rafts and nonraft membranes or under conditions where one or the other molecule partitions into a less favored membrane domain. CD45 is also reduced in density fivefold in the immunological synapse, but it is found in nearby endosomal compartments, which may afford it access to Lck (37). According to this model, access of CD45 (outside the rafts) to Lck (inside the rafts) will depend greatly on the stability of these membrane domains and the rate of transient movement of raft resident or excluded proteins into the adjacent domains. This issue is critical for understanding how the raft compartment works in signaling and is a matter of current debate: Is moving through a raft like more like getting stuck in quicksand or like walking through a revolving door?

Although Csk is a soluble kinase, its $\mathrm{SH} 2$ domain has recently been found to interact with the transmembrane adapter protein Csk-binding protein ( $\mathrm{Cbp}$, also known as PAG) $(38,39)$. Cbp includes a very small ectodomain and a large cytoplasmic domain containing palmitoylation sites and multiple phosphorylation sites. Its structure is very similar to that of LAT, and, like LAT, it is concentrated in rafts. However, while LAT predominantly recruits activating signaling molecules, $\mathrm{Cbp}$ recruits the negative regulator Csk. Csk is also found in a complex with the soluble protein tyrosine phosphatase PEP, which acts efficiently to dephosphorylate the Lck activation loop (40). Given the dual action of Csk and PEP, Cbp apparently recruits a potent Lck downregulation complex. The current working model is that activated Lck in the rafts phosphorylates $\mathrm{Cbp}$ and increases recruitment of Csk to the vicinity of the activated Lck (Figure 1). Thus, in the resting state Lck recruits its own silencer. Following activation, not only is Lck activity triggered, but $\mathrm{Cbp}$ becomes dephosphorylated by an as-yet unidentified phosphatase. These findings may in part explain the ability of cholesterol extraction to activate the kinase cascade in $\mathrm{T}$ cells in a transient manner, since dissolution of rafts will separate Lck from $\mathrm{Cbp} / \mathrm{Csk} / \mathrm{PEP}$ and permit it to interact with
CD45. Thus, it appears that rafts are centers of signaling in which balanced negative regulation can be reversed rapidly during TCR triggering.

\section{Keeping rafts apart}

Segregation of surface receptors is an important aspect of the immunological synapse and represents an important insight into the mechanisms of $\mathrm{T}$ cell regulation. In immunological synapse formation, not all the molecules that are engaged at the interface move to the same point in the synapse. In this regard, synapse formation is unlike the clustering seen when bivalent antibodies are used to "cap" surface proteins. In co-capping experiments, all the associated molecules appear to move to the same point, with no segregation of the various components. Thus, CD2 and LFA-1 will co-cap in antibody studies (41), but they remain segregated from one another in the immunological synapse and other cellcell interfaces (42). While LFA-1 is in communication with the TCR through a process of inside-out signaling (43), LFA-1 and the TCR are segregated in the immunological synapse $(14,16)$. This segregation is significant, because it places constraints on direct collaboration between LFA-1 and the TCR. For example, the adapter protein Fyb/SLAP-130 (see Leo et al., this Perspective series, ref. 44) associates physically both with the TCR and functionally with integrins such as LFA- $1(45,46)$. It links TCR signaling to LFA-1 activation $(47,48)$, but molecules of Fyb/SLAP-130 that interact with the TCR will be far from the site of LFA- 1 activation. Thus, active Fyb/SLAP-130 may have to diffuse across micrometers of cytoplasm to transduce a signal from TCR to LFA-1.

The basis of segregation of CD2-CD48 interactions from LFA-1-ICAM-1 interactions, and TCR-MHC-peptide interactions from LFA-1-ICAM-1 interactions, is
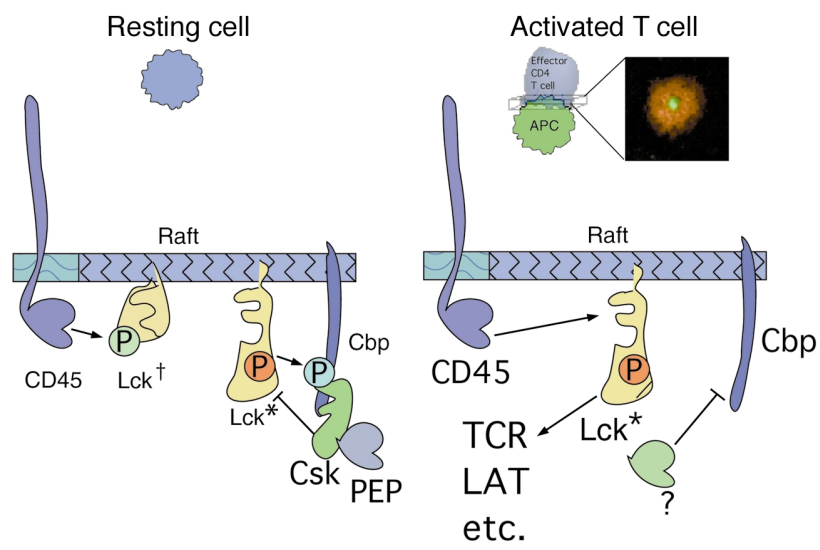

\section{Figure 1}

Regulation of Lck by rafts and Cbp. Cbp is localized to rafts in resting cells, where it is phosphorylated by active Lck*, an Src family PTK. This modification recruits Csk, which inactivates Lck ${ }^{\dagger}$ by phosphorylating its C-terminal regulatory site, and PEP, which dephosphorylates the Lck activation loop. Another phosphatase, CD45, is localized outside the rafts but may have access to Lck at the lateral boundary or during rare meetings due to partitioning into the same membrane domain. Activation of the TCR results in dephosphorylation of $\mathrm{Cbp}$ through the action of an unidentified phosphatase, thus removing the inhibitory complex containing Csk and PEP. 
a

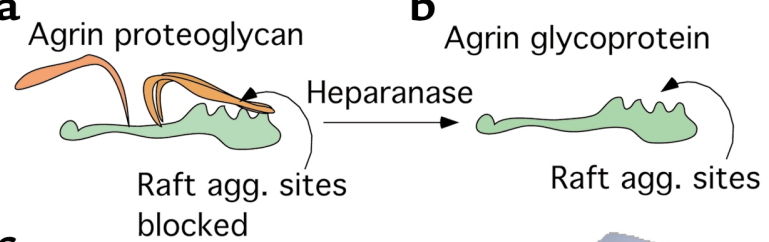

c
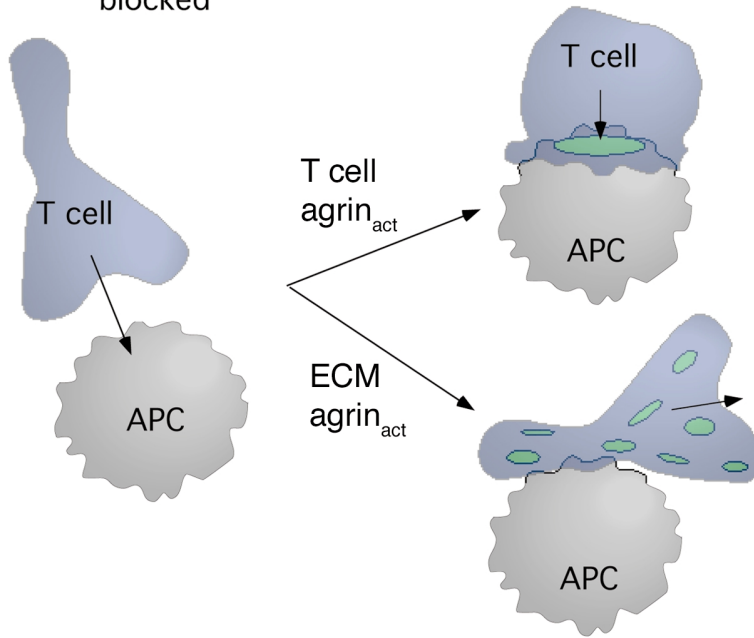

Figure 2

Hypothesis for agrin regulation of immunological synapse formation. (a) Raft-aggregating activity of agrin proteoglycan is regulated by intramolecular interaction with heparan sulfate chains. (b) When these chains are degraded by the lymphocyte heparanase, the raft-aggregating activity is expressed. (c) Recent evidence indicates that $\mathrm{T}$ cell agrin glycoprotein $\left(\right.$ agrin $_{\text {act }}$ ) enhances synapse formation and $T$ cell activation. Conversely, if agrin glycoprotein is prevented from entering the synapse, perhaps through an interaction with laminin or other ECM components, the formation of the synapse may be destabilized by ectopic raft aggregates, thus preventing $T$ cell activation. Thus, in the presence of specific ECM components, the effect of actin conversion from proteoglycan to glycoprotein may be inhibitory, whereas in an ECM-depleted site like a lymph node it may enhance responses.

likely to be the difference in size of these molecules. $\mathrm{T}$ cell CD2 and TCRs on the T cell hold CD48 and MHCpeptide, respectively, on the APC at an intermembrane distance of about $15 \mathrm{~nm}$. In contrast, LFA- 1 on the $\mathrm{T}$ cell holds ICAM- 1 on the APC at an intermembrane distance of about $40 \mathrm{~nm}$ (49). CD2 and TCR are of similar size, so they may be expected to cosegregate and cooperate. Consistent with this model, extended forms of the CD2 ligand CD48 on the APC not only fail to enhance but actually inhibit $\mathrm{T}$ cell activation (50).

The role of rafts in this physical segregation should also be considered. Activated TCRs translocate into rafts at least transiently, but the $\mathrm{CD} 28$ coreceptor, an important source of Signal 2, does not appear in biochemical analyses to be localized to rafts. However, it is clear that the CD28 and the TCR colocalize within the central cluster of the immunological synapse $(14,15)$. Therefore, it is possible that the central cluster of the immunological synapse is not homogenous but represents a mosaic of raft and nonraft membranes that cannot be resolved by light microscopy. This is a particularly important point, since the finding that $\mathrm{CD} 28$ can trigger recruitment of the ganglioside GM1 to sites of TCR engagement had originally lent support to the model that the center of the immunological synapse is highly enriched in raft membranes. Despite this enrichment, it now appears, the central cluster may include diverse membrane structures. Similarly, electron microscopy studies on the $\mathrm{F} c \varepsilon$ receptor $(\mathrm{Fc \varepsilon R})$ indicate that signaling may be focused in small but heterogeneous membrane domains (51). These regions, which contain the receptor itself, along with LAT and various Src and Syk family PTKs, are adjacent to clathrin-coated pits, to which the FceR is delivered along actin-rich tracks. Since clathrin-coated pits form on nonraft membranes (52), it is likely that the LAT-enriched regions are rafts that are surrounded by nonraft membranes, decorated with clathrin. Even under the conditions in which the electron micrographic study was done, which favored raft clustering, local membranes appeared as a patchwork, with significant interfaces between domains and evidence for specific transport paths between raft and nonraft regions. Such heterogeneity is consistent with the active endocytosis and exocytosis known to occur in the central region of the immunological synapse. The diverse membrane structures in the central region likely serve multiple functions in TCR signaling.

\section{The functional significance of raft dynamics}

The idea that receptor or raft rearrangement will be important for signaling suggests that regulating this process will ultimately affect the immune response, as has been suggested to explain suppression of immune responses by the bivalent lectin galectin-3 (53). Mgat-5-deficient mice, which lack a carbohydrate modification that generates the ligand for galectin-3, are susceptible to autoimmune disease and are hyperresponsive to a variety of stimuli. They also show enhanced raft accumulation at sites of activation. Remarkably, this phenotype can be mimicked in normal $\mathrm{T}$ cells by adding a simple sugar inhibitor of galectin-3 binding, suggesting that galectin- 3 forces associations between surface molecules that inhibit the molecular rearrangement required for effective immunological synapse formation. It remains uncertain whether this interference affects Signal 1 (TCR interaction), Signal 2 (costimulation), or both, but it has been suggested that raft movement contributes to Signal 2 and that galectin- 3 specifically opposes costimulatory signals. The absence of such signals in thymi of Mgat-5-deficient animals would then erode the margin of safety between thymocytes and mature $\mathrm{T}$ cell activation, perhaps accounting for their autoimmune disease.

Agrin is a high-molecular weight component of ECM whose best-known role is in aggregation of the acetylcholine receptor in the neuromuscular junction (NMJ). This function, which is mediated by the receptor type PTK Musk (54), appears to be quite specific to the nervous system, since spliced forms of Musk that are required in this response are highly motor neuron-specific and have no obvious counterparts in the immune system. Agrin, conversely, is a large multidomain protein expressed in multiple tissues, where it probably acts in varied roles (55). A modified form of agrin associated with splenic lymphocytes 
promotes $\mathrm{T}$ cell activation (56) and has been shown to drive the clustering of lipid rafts and surface molecules on these cells. Significantly, this protein, but not the agrin forms found in the NMJ or elsewhere, also favors the antigen-specific activation of $\mathrm{T}$ cells from TCR transgenic mice. The active form of agrin is produced posttranslationally in activated lymphocytes. Its molecular weight is consistent with removal of the heparan sulfate chains to convert it from a proteoglycan into a conventional glycoprotein (57), presumably mediated by heparanase produced by activated lymphocytes $(58,59)$.

The simplest model for agrin function holds that, like galectin-3, the agrin glycoprotein binds specifically to some raft constituent. Unlike the galectin, however, agrin may directly aggregate rafts in the immunological synapse to promote $T$ cell activation. The heparan chains of the agrin proteoglycan may mask these active sites such that only the agrin glycoprotein without the heparan sulfate chains will be active in raft clustering. $\mathrm{T}$ cell-derived heparanase is likely to modify both the $T$ cell-derived and ECM forms of agrin to convert them to the active glycoprotein form. In the hypothetical model shown in Figure 2, the effect of the resulting agrin glycoproteins may be opposite, depending upon the relative location. Soluble agrin glycoprotein and agrin glycoprotein associated with the $T$ cell surface may promote raft clustering the synapse. In contrast, agrin glycoproteins in the ECM may inhibit $\mathrm{T}$ cell activation by inducing ectopic raft clusters that cannot be translocated into the synapse.

Thus, like galectin-3, agrin is a new candidate immunomodulator in the ECM that may differentially regulate regional immunity and autoimmune responses. Testing these ideas will be easiest in mouse model systems, although the agrin-deficient mouse is perinatal-lethal, so the generation of chimeric mice or conditional knockouts will be necessary (60).

\section{Conclusions}

The immunological synapse and membrane rafts reflect two levels of resolution in our view of $T$ cell activation. The initial and highly productive focus of the field has been on biochemistry with its molecularlevel detail and the ability to identify the critical molecules and test their role in a second level of genetic analysis. Over the last two years, there has been an increasing attention to the compartmentalization of signaling in lymphocyte activation. The immunological synapse is a micron-scale structure that is readily followed with a light microscope. Therefore, significant progress should be made in the near future on the genetics of immunological synapse formation and the importance of this process in a number of normal and pathological situations. On the other hand, rafts are submicroscopic structures that really cannot be resolved by light microscopy. Even when we can see them, for example, following receptor aggregation, they are probably not concentrated to the point of homogeneity. The well-defined clusters of the immunological synapse are likewise probably a mosaic of different membrane domains.
Techniques to bridge the resolution gap and provide a continuous line of experimental observations from the molecular to the cellular level are emerging, and more progress is predicted. Nonetheless, recent studies linking biochemistry, membrane structure, and the supramolecular organization of the immunological synapse, or simpler membrane caps, have provided a number of important new concepts for immunobiology, including the potential importance of manipulating immunological synapse formation and $\mathrm{T}$ cell activation with soluble raft-binding proteins. Even in the resting cell, where rafts are homogenously distributed, the action of these compartments is evident in the signaling homeostasis that maintains $T$ cell survival and leaves quiescent $\mathrm{T}$ cells poised in a state of Zen balance - resting but ready.

\section{Acknowledgments}

I thank Richard Burack, Rajat Varma, and Michael Edidin for discussions on the nature of rafts that influenced the ideas in this review. I thank Roxanne Barrett for preparation of the manuscript. My work is supported by the NIH and by an Irene Diamond Professorship in Immunology.

1. Katz, J.D., Wang, B., Haskins, K., Benoist, C., and Mathis, D. 1993. Following a diabetagenic $\mathrm{T}$ cell from genesis through pathogenesis. Cell. 74:1089-1100

2. Lafaille, J.J., Nagashima, K., Katsuki, M., and Tonegawa, S. 1994. High incidence of spontaneous autoimmune encephalomyelitis in immunodeficient anti-myelin basic protein $\mathrm{T}$ cell receptor transgenic mice. Cell. 78:399-408.

3. Peterson, D.A., DiPaolo, R.J., Kanagawa, O., and Unanue, E.R. 1999. Negative selection of immature thymocytes by a few peptide-MHC complexes: differential sensitivity of immature and mature $\mathrm{T}$ cells. $J$. Immunol. 162:3117-3120.

4. Alam, S.M., et al. 1996. T-cell-receptor affinity and thymocyte positive selection. Nature. 381:616-620.

5. Williams, C.B., Engle, D.L., Kersh, G.J., Michael White, J., and Allen, P.M. 1999. A kinetic threshold between negative and positive selection based on the longevity of the T cell receptor-ligand complex. J. Exp. Med. 189:1531-1544.

6. Kaech, S.M., and Ahmed, R. 2001. Memory CD8+ T cell differentiation: initial antigen encounter triggers a developmental program in naive cells. Nat. Immunol. 2:415-422.

7. Wong, P., and Pamer, E.G. 2001. Cutting edge: antigen-independent CD8 T cell proliferation. J. Immunol. 166:5864-5868.

8. Iezzi, G., Karjalainen, K., and Lanzavecchia, A. 1998. The duration of antigenic stimulation determines the fate of naive and effector $T$ cells. Immunity. 8:89-95.

9. Jenkins, M.K., and Schwartz, R.H. 1987. Antigen presentation by chemically modified splenocytes induces antigen-specific T cell unresponsiveness in vitro and in vivo. J. Exp. Med. 165:302-319.

10. Unanue, E.R. 1984. Antigen-presenting function of the macrophage. Annu. Rev. Immunol. 2:395-428.

11. Shahinian, A., et al. 1993. Differential T cell costimulatory requirements in CD28-deficient mice. Science. 261:609-612.

12. Curtsinger, J.M., et al. 1999. Inflammatory cytokines provide a third signal for activation of naive CD4+ and CD8+ T cells. J. Immunol. 162:3256-3262.

13. Purves, D., and Lichtman, J.W. 1985. Principles of neural development. Sinauer Associates Inc. Sunderland, Massachusetts, USA. 433 pp.

14. Grakoui, A., et al. 1999. The immunological synapse: a molecular machine controlling T cell activation. Science. 285:221-227.

15. Bromley, S.K., et al. 2001. The immunological synapse. Annu. Rev. Immunol. 19:375-396

16. Monks, C.R., Freiberg, B.A., Kupfer, H., Sciaky, N., and Kupfer, A. 1998. Three-dimensional segregation of supramolecular activation clusters in T cells. Nature. 395:82-86.

17. Turley, S.J., et al. 2000. Transport of peptide-MHC class II complexes in developing dendritic cells. Science. 288:522-527.

18. Dustin, M.L., and Cooper, J.A. 2000. The immunological synapse and the actin cytoskeleton: molecular hardware for T cell signaling. Nat. Immunol. 1:23-29.

19. Qi, S.Y., Groves, J.T., and Chakraborty, A.K. 2001. Synaptic pattern for- 
mation during cellular recognition. Proc. Natl. Acad. Sci. USA. 98:6548-6553

20. Wülfing, C., and Davis, M.M. 1998. A receptor/cytoskeletal movement triggered by costimulation during $\mathrm{T}$ cell activation. Science. 282:2266-2269.

21. Klausner, R.D., Kleinfeld, A.M., Hoover, R.L., and Karnovsky, M.J. 1980 Lipid domains in membranes. Evidence derived from structural perturbations induced by free fatty acids and lifetime heterogeneity analysis. J. Biol. Chem. 255:1286-1295.

22. Stefanova, I., Horejsi, V., Ansotegui, I.J., Knapp, W., and Stockinger, H. 1991. GPI-anchored cell-surface molecules complexed to protein tyrosine kinases. Science. 254:1016-1019.

23. Brown, D.A., and London, E. 1998. Functions of lipid rafts in biological membranes. Annu. Rev. Cell Dev. Biol. 14:111-136.

24. Xavier, R., Brennan, T., Li, Q., McCormack, C., and Seed, B. 1998. Membrane compartmentation is required for efficient $\mathrm{T}$ cell activation. Immunity. 8:723-732.

25. Kabouridis, P.S., Janzen, J., Magee, A.L., and Ley, S.C. 2000. Cholesterol depletion disrupts lipid rafts and modulates the activity of multiple signaling pathways in T lymphocytes. Eur. J. Immunol. 30:954-963.

26. Weiss, A., and Littman, D.R. 1994. Signal transduction by lymphocyte antigen receptors. Cell. 76:263-274.

27. Dustin, M.L., and Chan, A.C. 2000. Signaling takes shape in the immune system. Cell. 103:283-294.

28. Billadeau, D.D., and Leibson, P.J. 2002. ITAMs versus ITIMs: striking a balance during cell regulation. J. Clin. Invest. 109:161-168. DOI:10.1172/JCI200214843.

29. Zhang, W., Sloan-Lancaster, J., Kitchen, J., Trible, R.P., and Samelson, L.E. 1998. LAT: the ZAP-70 tyrosine kinase substrate that links T cell receptor to cellular activation. Cell. 92:83-92.

30. Moarefi, I., et al. 1997. Activation of the Src-family tyrosine kinase Hck by SH3 domain displacement. Nature. 385:650-653.

31. Sicheri, F., Moarefi, I., and Kuriyan, J. 1997. Crystal structure of the Src family kinase Hck. Nature. 385:602-609.

32. Hermiston, M.L., Xu, Z., Majeti, R., and Weiss, A. 2002. Reciprocal regulation of lymphocyte activation by tyrosine kinases and phosphatases. $J$. Clin. Invest. 109:9-14. DOI:10.1172/JCI200214794.

33. Bergman, M., et al. 1992. The human p50csk tyrosine kinase phosphorylates p56lck at Tyr-505 and down regulates its catalytic activity. EMBO J. 11:2919-2924.

34. Ostergaard, H.L., et al. 1989. Expression of CD45 alters phosphorylation of the lck-encoded tyrosine protein kinase in murine lymphoma T-cell lines. Proc. Natl. Acad. Sci. USA. 86:8959-8963.

35. Yanagi, S., et al. 1996. CD45 modulates phosphorylation of both autophosphorylation and negative regulatory tyrosines of Lyn in B cells. J. Biol. Chem. 271:30487-30492.

36. D'Oro, U., and Ashwell, J.D. 1999. Cutting edge: the CD45 tyrosine phosphatase is an inhibitor of Lck activity in thymocytes. J. Immunol. 162:1879-1883.

37. Johnson, K.G., Bromley, S.K., Dustin, M.L., and Thomas, M.L. 2000. A supramolecular basis for CD45 regulation during T cell activation. Proc. Natl. Acad. Sci. USA. 97:10138-10143.

38. Kawabuchi, M., et al. 2000. Transmembrane phosphoprotein Cbp regulates the activities of Src-family tyrosine kinases. Nature. 404:999-1003.

39. Brdicka, T., et al. 2000. Phosphoprotein associated with glycosphingolipid-enriched microdomains (PAG), a novel ubiquitously expressed transmembrane adapter protein, binds the protein tyrosine kinase csk and is involved in regulation of $\mathrm{T}$ cell activation. J. Exp. Med. 191:1591-1604

40. Cloutier, J.F., and Veillette, A. 1999. Cooperative inhibition of T-cell antigen receptor signaling by a complex between a kinase and a phosphatase. J. Exp. Med. 189:111-121.

41. Rosenman, S.J., Ganji, A.A., Tedder, T.F., and Gallatin, W.M. 1993. Syncapping of human $\mathrm{T}$ lymphocyte adhesion/activation molecules and their redistribution during interaction with endothelial cells. J. Lenkoc. Biol. 53:1-10.

42. Dustin, M.L., et al. 1998. A novel adapter protein orchestrates receptor patterning and cytoskeletal polarity in T cell contacts. Cell. 94:667-677.

43. Dustin, M.L., and Springer, T.A. 1989. T cell receptor cross-linking transiently stimulates adhesiveness through LFA-1. Nature. 341:619-624

44. Leo, A., Wienands, J., Baier, G., Horejsi, V., and Schraven, B. 2002. Adapters in lymphocyte signaling. J. Clin. Invest. In press. DOI:10.1172/JCI200214942.

45. Krause, M., et al. 2000. Fyn-binding protein (Fyb)/SLP-76-associated protein (SLAP), Ena/vaso3dilator-stimulated prosphoprotein (VASP) proteins and the Arp $2 / 3$ complex link T cell receptor (TCR) signaling to the actin cytoskeleton. J. Cell Biol. 149:181-194.

46. Hunter, A.J., Ottoson, N., Boerth, N., Koretzky, G.A., and Shimizu, Y. 2000. Cutting edge: a novel function for the SLAP-130/FYB adapter protein in beta 1 integrin signaling and T lymphocyte migration. J. Immunol. 164:1143-1147.

47. Peterson, E.J., et al. 2001. Coupling of the TCR to integrin activation by Slap-130/Fyb. Science. 293:2263-2265.

48. Griffiths, E.K., et al. 2001. Positive regulation of T cell activation and integrin adhesion by the adapter Fyb/Slap. Science. 293:2260-2263.

49. Springer, T.A. 1990. Adhesion receptors of the immune system. Nature. 346:425-433

50. Wild, M.K., et al. 1999. Dependence of T cell antigen recognition on the dimensions of an accessory receptor-ligand complex. J. Exp. Med. 190:31-41.

51. Wilson, B.S., Pfeiffer, J.R., and Oliver, J.M. 2000. Observing FcepsilonRI signaling from the inside of the mast cell membrane. J. Cell Biol. 149:1131-1142.

52. Nichols, B.J., et al. 2001. Rapid cycling of lipid raft markers between the cell surface and Golgi complex. J. Cell Biol. 153:529-541.

53. Demetriou, M., Granovsky, M., Quaggin, S., and Dennis, J.W. 2001. Negative regulation of T-cell activation and autoimmunity by Mgat5 N-glycosylation. Nature. 409:733-739.

54. Glass, D.J., et al. 1996. Agrin acts via a MuSK receptor complex. Cell. 85:513-523.

55. Gesemann, M., et al. 1996. Alternative splicing of agrin alters its binding to heparin, dystroglycan, and the putative agrin receptor. Neuron. 16:755-767.

56. Khan, A.A., Bose, C., Yam, L.S., Soloski, M.J., and Rupp, F. 2001. Physiological regulation of the immunological synapse by agrin. Science. 292:1681-1686

57. Tsen, G., Halfter, W., Kroger, S., and Cole, G.J. 1995. Agrin is a heparan sulfate proteoglycan. J. Biol. Chem. 270:3392-3399.

58. Parish, C.R., Freeman, C., and Hulett, M.D. 2001. Heparanase: a key enzyme involved in cell invasion. Biochim. Biophys. Acta. 1471:M99-M108.

59. Fridman, R., et al. 1987. Soluble antigen induces $\mathrm{T}$ lymphocytes to secrete an endoglycosidase that degrades the heparan sulfate moiety of subendothelial extracellular matrix. J. Cell. Physiol. 130:85-92.

60. Gautam, M., et al. 1996. Defective neuromuscular synaptogenesis in agrin-deficient mutant mice. Cell. 85:525-535. 